\title{
Ex Vivo Comparative Study of the Effect of Different Concentrations of Green Tea Extract and Two Common Irrigants on Root Canals In- fected with Enterococcus faecalis
}

\author{
B Dadresanfar ${ }^{1}$, M Vatanpour ${ }^{1}$, M Farahmand ${ }^{* 2}$, S Taheri ${ }^{3}$, HR Mahaseni \\ Aghdam $^{4}$
}

1-Assistant Professor, Endodontics Dept, Faculty of Dentistry, Tehran Medical Sciences, Islamic Azad University, Tehran, Iran 2-Postgraduate student, Orthodontics Dept, Faculty of Dentistry, Tehran Medical Sciences, Islamic Azad University, Tehran, Iran 3-Dentist, Frankfurt, Germany

4-Assistant professor, Oral and Maxillofacial Surgery and Implant Research Center, Dental Faculty, Tehran Medical Sciences, Islamic Azad University, Tehran, Iran

ARTICLE INFO
Article History
Received: Nov 2018
Accepted: Dec 2018
ePublished: Jan 2019

Corresponding author:

M Farahmand, Postgraduate student, Orthodontics Dept, Faculty of Dentistry, Tehran Medical Sciences, Islamic Azad University, Tehran, Iran

\begin{abstract}
Background and Aim: One of the main goals of endodontic treatments is to disinfect the root canal and dentin tubules. This study compared the antimicrobial effect of different concentrations of green tea extract with that of two common irrigants on Enterococcus faecalis (E. faecalis) in the root canal system.

Materials and Methods: In this experimental study on 124 single canal teeth, suspensions derived from the 24-hour culture of E. faecalis were inoculated into the canals, and the samples were incubated for two weeks. Then, the teeth were divided into six experimental groups $(n=20)$ and two control groups $(n=2)$. In the first group, $5 \%$ sodium hypochlorite $(\mathrm{NaOCl})$, in the second group, $2 \%$ chlorhexidine gluconate $(\mathrm{CHX})$, in the third group, $3.125 \%$ green tea extract, in the fourth group, $12.5 \%$ green tea extract, in the fifth group, $25 \%$ green tea extract, and in the sixth group, normal saline was used for root canal irrigation. The next day, the extracted liquid from vortexed dentin fragments was cultured, and the colony-forming units (CFU) were counted 48 hours later. Data were analyzed using Mann-U-Whitney test.

Results: The CFU count for $\mathrm{NaOCl}$ and $\mathrm{CHX}$ showed a statistically significant difference compared to different groups of green tea extract $(\mathrm{P}<0.001)$. The percentage of microorganism reduction was $100 \%$ with $\mathrm{NaOCl}, 98.9 \%$ with $\mathrm{CHX}, 58.35 \%$ with $3.125 \%$ green tea, $8.1 \%$ with $12.5 \%$ green tea, $94.8 \%$ with $25 \%$ green tea, and $57.5 \%$ with normal saline.

Conclusion: The results of this study showed that green tea extract can be used in endodontic treatment as the final root canal irrigant considering its naturalness and its antimicrobial ability.

Keywords: Root Canal Irrigants, green tea extract polyphenon E, Enterococcus faecalis, Sodium Hypochlorite, Chlorhexidine
\end{abstract}

J Res Dent maxillofac Sci. 2019;4(2):32-40 DOI: 10.29252/jrdms.4.2.32

Introduction:

One of the major causes of root canal therapy (RCT) failure is the persistence of Enterococcus faecalis (E. Faecalis) in the canal. ${ }^{(1)}$ In one study, negative culture at the time of root filling was associated with $94 \%$ healing, whereas positive culture at the time of filling resulted in only $68 \%$ healing; thus, repair and restoration of the the periodontium in cases where the canals have been filled in the presence of microbial contamination have a low success rate. ${ }^{(2)}$

Research has shown that $\mathrm{E}$. faecalis is the most common resistant gram-positive anaerobic bacterium found in teeth with failed root canal treatment; this is due to the inherent resistance of this bacterium to antimicrobial agents, its and 
biofilm formation ability. ${ }^{(3)}$

Nowadays, various antimicrobial agents are used for root canal irrigation, including MTAD, $2 \%$ chlorhexidine (CHX) gel, 5\% sodium hypochlorite $(\mathrm{NaOCl})$, some of which have toxic effects in addition to antimicrobial properties. ${ }^{(4,5)}$ If intra-canal microorganisms, especially E. faecalis, are not completely eliminated, the chances of treatment failure will be high, leading to the formation of periradicular lesions and, in more severe cases, tooth loss due to the progression of the lesion. ${ }^{(1)}$

One of the research priorities is to find a material that, while having antimicrobial activity, has better biocompatibility. In this study, the use of essential oil of green tea as an intra-canal irrigant was considered. In-vitro antimicrobial effects of this substance have been studied and proven. ${ }^{(6,7)}$

Also, its antimicrobial effect on intra-canal microorganisms, especially on E. faecalis, has been proven. However, its most effective concentration compared to common root canal irrigants has not been investigated. ${ }^{(7)}$ Considering this information gap and inconsistencies in previous research on root canal irrigants, the purpose of this study was to compare the antimicrobial effect of different concentrations of green tea extract with that of two common root canal irrigants on E. faecalis in the root canal system (ex-vivo) at the Dental Faculty of Islamic Azad University of Medical Sciences, Tehran, Iran, in 2012-13.

\section{Materials and Methods}

In this experimental study, 150 extracted single-rooted and single canal human teeth without any internal or external resorption, calcification, crack, and decay at root surface and with closed apices were collected from the clinics in Tehran city. The teeth were kept in normal saline until preparation.

The teeth were cleaned of soft tissue debris using scaling curettes, and then, using a highspeed handpiece fitted with a fissure bur (Tizkavan, Tehran, Iran), after caries removal, an access cavity was prepared with direct access to the root canal. Then, teeth with a 16-mm working length were selected. Finally, 124 teeth were selected for the next steps. The samples were prepared by a rotary system using ProTaper files (ProTaper Universal, Dentsply Maillefer, Switzerland).

To remove the smear layer and to keep the dentinal tubules open for bacterial infiltration, the teeth were first treated with 17\% Ethylenediaminetetraacetic acid (EDTA) for one minute, and then, with $5.25 \% \mathrm{NaOCl}$ for 4 minutes, and finally with sterile distilled water for 10 minutes.

The apical foramen of each tooth was sealed by light-cure glass-ionomer (GC Corp., Tokyo, Japan). To prevent external bacterial infiltration, the outer surface of the teeth was first coated with two layers of colorless nail polish and later with two coats of colored nail polish.

Determination of minimum inhibitory concentration (MIC) of green tea extract and preparation of different concentrations:

The serial dilution method was used to determine the MIC of green tea extract. For this purpose, a solution at the dilution of (distilled water) was first prepared, and then, 10 sterile test tubes were prepared and numbered .

First, $0.5 \mathrm{cc}$ of Tryptic Soy Broth (TSB; Merck, Darmstadt, Germany) was added to the tubes, and then, $0.5 \mathrm{cc}$ of the previously prepared $5 \mathrm{mg} /$ $\mathrm{ml}$ dilution was added to the first tube. Then, we removed $1 / 2$ of the first tube and added it to the second tube (1/4), and we continued the dilution process to the tenth tube.

Finally, we added 20 or $20 \mu$ of the microorganism to all tubes (microorganism preparation: we dissolved some of the microorganisms in some TSB medium and compared the turbidity to $0.5 \mathrm{McFarland}$ standard, which was measured with a spectrophotometer to be equal to 0.08 0.13 . We added enough microorganisms to reach 0.5 McFarland standard turbidity.).

All the tubes were incubated at $37^{\circ} \mathrm{C}$ for 24 hours. Afterward, 0.05-ml samples were collected with a standard platinum microbiology loop, which were then cultured in the bile esculin agar medium (V44247, the specific medium of E. faecalis; Merck, Darmstadt, Germany). Finally, the $1 / 16$ dilution equivalent to $31.25 \mathrm{mg} / \mathrm{ml}(3.125 \%)$ was designated as the MIC (Figure 1). 


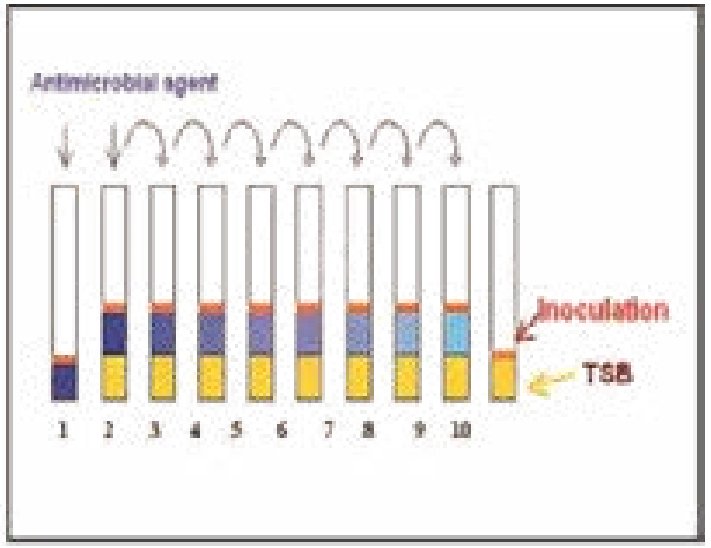

Figure 1. Determination of minimum inhibitory concentration (MIC); TSB=Tryptic Soy Broth

Three concentrations of $3.125 \%, 12.5 \%$, and $25 \%$ were prepared accordingly (Figure 2 ).

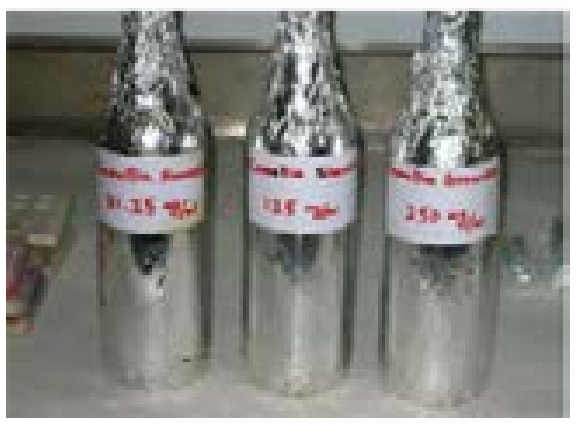

Figure 2. Different concentrations of green tea extract

Inoculation of $\mathrm{E}$. faecalis in dental canals: The samples were placed in sterile microtubes (sterile Cryotubes with screw cap, Cryo.s, Greiner Bio-One GmbH, Frichenhausen, Germany) containing TSB (a nutrient medium for bacterial growth selected according to previous studies) and sterile solution, which were autoclaved at $121^{\circ} \mathrm{C}$ and pressure of 15 Psi for 30 minutes. Then, to ensure sterilization, the specimens were incubated at $37^{\circ} \mathrm{C}$ for 48 hours to be sterilized again if turbidity was observed in the TSB medium. Next, $0.02 \mathrm{ml}$ of the 24-hour culture of pure bacterial suspension of $\mathrm{E}$. this time.
Final irrigation:

The samples were divided into six groups of 20 samples each and were tested as follows:

Before rinsing, dental canals were evacuated from the liquid TSB medium inside the canals using \#30 sterile paper points (Tianjin Gapadent Co. Ltd., China).

(A) In the first group, 20 teeth were washed with $10 \mathrm{cc}$ of $5 \% \mathrm{NaOCl}$ (Clorox) with a 2-cc syringe and 27-gauge needles.

B) In the second group, 20 teeth were washed with $10 \mathrm{cc}$ of $2 \% \mathrm{CHX}$ (FGM, Portugal) with a 2-cc syringe and 27-gauge needle heads.

C) In the third group, 20 teeth were washed with $10 \mathrm{cc}$ of green tea extract (3.125\%; SohaJissa Co., Mazandaran, Iran) with a 2-cc syringe and 27-gauge needles.

D) In the fourth group, 20 teeth were washed with $10 \mathrm{cc}$ of green tea extract (12.5\%; SohaJissa Co., Mazandaran, Iran) with a 2-cc syringe and 27-gauge needles.

E) In the fifth group, 20 teeth were rinsed with $10 \mathrm{cc}$ of green tea extract (25\%; by Soha-Jissa Co., Mazandaran, Iran) with a 2-cc syringe and 27-gauge needles.

F) In the sixth group, 20 teeth were washed with $10 \mathrm{cc}$ of normal saline with a 2-cc syringe and 27-gauge needles.

In the positive control group, the samples did not receive any irrigation after the inoculation of microorganisms.

In the negative control group, the samples were sterilized only.

It should be noted that all irrigants were left in all root canals for 10 minutes, and then, the root canals were dried with paper points, and the teeth were placed in sterile microtubes containing $0.02 \mathrm{ml}$ of sterile TSB.

Sampling of root canals and microbiological tests:Dental canal biopsy was postponed to the next day. To keep the remaining bacteria alive after the intervention, sterile TSB fluid (equal to the volume of each root canal, $0.02 \mathrm{ml}$ on average) was added to the canals using a 5-cc sterile syringe (18 gauge).

Twenty-four hours later, the TSB fluid was first removed from the canals using sterile paper points, and sterile saline (equal to the volume of each root canal) was added to each canal using a 5-cc sterile syringe (18 gauge). 
After pouring the sterile serum, each canal was circumferentially filed for 20 seconds using a sterile Hedstrom file (Mani Inc., Tochigi, Japan). The file, along with sterile paper points used to collect the serum in the canal - containing dentin fragments and possibly viable bacteria - was transferred into a test tube containing $10 \mathrm{cc}$ of sterile normal saline, and the dilution process was carried out to count the colonies.

The test tubes were vortexed (Cenco Instrumenten, Breda, The Netherlands) for 20 seconds, and thus, the possibly viable bacteria were separated from the files and the paper points.

In the next step, from the vortexed solution, $0.05-$ $\mathrm{ml}$ samples were collected (using a standard platinum inoculation loop with the ability to harvest 50 $\lambda$ of microbes) and were cultured in the bile esculin agar medium. Positive and negative control samples were also taken and cultured in the bile esculin agar medium.

Colonies were counted 48 hours later (Figure 3)

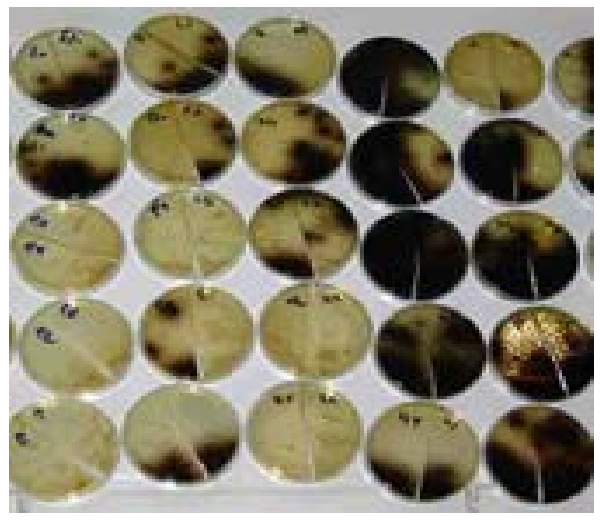

\section{Figure 3. 48-hour microbial culture after sampling}

by a special counting machine (Funke-Gerber, Germany), and $\mathrm{CFU} / \mathrm{ml}$ was calculated; logarithm was taken to reduce data dispersion.

The calculated CFU was also divided into five grades according to the microbiology standard as follows:

$1=$ low $(1-10000 \mathrm{CFU} / \mathrm{ml})$

$2=$ low moderate $(10000-20000 \mathrm{CFU} / \mathrm{ml})$

$3=$ moderate $(20000-50000 \mathrm{CFU} / \mathrm{ml})$

$4=$ moderate high $(50000-100000 \mathrm{CFU} / \mathrm{ml})$

$5=$ high $(>100,000 \mathrm{CFU} / \mathrm{ml})$, referred to as $\mathrm{CFU}$ grade.
The data were initially analyzed by Kolmogorov-Smirnov statistical test, and the distribution of data was not normal $(\mathrm{P}>0.05)$. Therefore, data were analyzed by Kruskal-Wallis statistical test. This test showed a statistically significant difference in comparing the mean CFU grades of the six studied groups $(\mathrm{P}=0.001)$. Therefore, the groups were analyzed using Mann-U-Whitney statistical test. Alpha $(\alpha)$ coefficient adjustment was performed with the Bonferroni adjustment method to maintain the type 1 error rate of $5 \%$.

\section{Results:}

The results of the treatment on the subject groups are presented in Table 1.

Table 1: Distribution of dental samples by the number of bacterial colonies in the study groups

\begin{tabular}{|c|c|c|}
\hline $\mathrm{CFU} / \mathrm{ml}$ & Mean \pm SE & $\begin{array}{c}\text { Kruskal-Wallis } \\
\text { (P-value) }\end{array}$ \\
\hline Treatment with $5 \% \mathrm{NaOCl}$ & 0.000 & \multirow{6}{*}{0.001} \\
\hline Treatment with $2 \% \mathrm{CHX}$ & $1100 \pm 458.25$ & \\
\hline $\begin{array}{l}\text { Treatment with } 3.125 \% \\
\text { green tea extract }\end{array}$ & $41600 \pm 9919.22$ & \\
\hline $\begin{array}{l}\text { Treatment with } 12.5 \% \\
\text { green tea extract }\end{array}$ & $19000 \pm 4046.44$ & \\
\hline $\begin{array}{l}\text { Treatment with } 25 \% \text { green } \\
\text { tea extract }\end{array}$ & $5200 \pm 1157.58$ & \\
\hline $\begin{array}{l}\text { Treatment with normal } \\
\text { saline }\end{array}$ & $43000 \pm 7419.74$ & \\
\hline
\end{tabular}

$\mathrm{SE}=$ Standard Error, $\mathrm{CFU}=$ Colony-forming unit, $\mathrm{NaOCl}=$ Sodium hypochlorite, $\mathrm{CHX}=$ Chlorhexidine

According to Kruskal-Wallis test, there was a significant difference between group $1(5 \% \mathrm{NaOCl})$ and group $2(2 \% \mathrm{CHX})$ for $\mathrm{CFU}(\mathrm{P}<0.05)$, but for $\mathrm{CFU}$ grade, there was no significant difference $(\mathrm{P}>0.05)$. There was a significant difference between group $1(5 \% \mathrm{NaOCl})$ and group 3 (3.125\% green tea extract) for CFU and CFU grade $(\mathrm{P}<0.001)$.

There was a significant difference between group $1(5 \% \mathrm{NaOCl})$ and group $4(12.5 \%$ green tea extract) for CFU and CFU grade $(\mathrm{P}<0.001)$. There was a significant difference between group $1(5 \% \mathrm{NaOCl})$ and group $5(25 \%$ green tea extract) for CFU and CFU grade $(\mathrm{P}<0.001$ and 0.03 , respectively).

There was a significant difference between group $1(5 \% \mathrm{NaOCl})$ and group 6 (normal saline) for $\mathrm{CFU}$ and $\mathrm{CFU}$ grade $(\mathrm{P}<0.001)$.

There was a significant difference between 
group $2(2 \% \mathrm{CHX})$ and group $3(3.125 \%$ green tea extract) for CFU and CFU grade $(\mathrm{P}<0.001)$.

There was a significant difference between group $2(2 \% \mathrm{CHX})$ and group $4(12.5 \%$ green tea extract) for CFU and CFU grade $(\mathrm{P}<0.001)$.

There was a significant difference between group 2 (2\% CHX) and group 5 (25\% green tea extract) for $\mathrm{CFU}$ and $\mathrm{CFU}$ grade $(\mathrm{P}=0.01$ and 0.037 , respectively).

There was a significant difference between group 2 (2\% CHX) and group 6 (normal saline) for $\mathrm{CFU}$ and $\mathrm{CFU}$ grade $(\mathrm{P}<0.001)$.

There was no significant difference between group $3(3.125 \%$ green tea extract) and group $4(12.5 \%$ green tea extract) for CFU and CFU grade $(\mathrm{P}>0.05)$.

There was a significant difference between group 3 (3.125\% green tea extract) and group 5 (25\% green tea extract) for CFU and CFU grade ( $\mathrm{P}<0.001$ and 0.001 , respectively).

There was no significant difference between group 3 (3.125\% green tea extract) and group 6 (normal saline) for CFU and CFU grade $(\mathrm{P}>0.05)$. There was a significant difference between group $4(12.5 \%$ green tea extract) and group 5 (25\% green tea extract) for CFU and CFU grade $(\mathrm{P}<0.002$ and 0.005 , respectively).

There was a significant difference between group $4(12.5 \%$ green tea extract) and group 6 (normal saline) for $\mathrm{CFU}$ and $\mathrm{CFU}$ grade $(\mathrm{P}=0.033$ and 0.023 , respectively).

There was a significant difference between group 5 (25\% green tea extract) and group 6 (normal saline) for $\mathrm{CFU}$ and $\mathrm{CFU}$ grade $(\mathrm{P}=0.001$ and $<0.001$, respectively).

\section{Discussion:}

This study was performed on 124 human singlerooted and single-canal posterior teeth to compare the antimicrobial effect of different concentrations of green tea extract with that of two common irrigants on E. faecalis in root canal system (in vitro). The results showed the reduction in the number of microorganisms to be $100 \%$ with $\mathrm{NaOCl}, 98.9 \%$ with CHX, $58.35 \%$ with $3.125 \%$ green tea, $81 \%$ with $12.5 \%$ green tea, $94.8 \%$ with $25 \%$ green tea, and $57.5 \%$ with normal saline.

Microorganisms have been long considered as a major cause of the progression of pulpal and peri- apical lesions. ${ }^{(8)}$ The purpose of root canal therapy is to remove microorganisms from the root canal system and to prevent reinfection. ${ }^{(9)}$ The number of anaerobic microorganisms increases if the root canal system remains infected for a long time. (7) E. faecalis is the most common gram-positive anaerobic bacterium that has a major role in the persistence of periradicular lesions after root canal treatment. ${ }^{(3,10)}$ It can withstand adverse environmental conditions. ${ }^{(11)}$ Also, its ability to form biofilms on root canal walls makes this bacterium 1000 times more resistant to antibodies, phagocytosis, and antimicrobial agents than other bacteria lacking the ability to form biofilms. ${ }^{(12)}$

Recently, due to the collection of E. faecalis from the root canal system of teeth with resistant and permanent infection, we are seeing increased research on this bacterium, ${ }^{(7,9)}$ and this microorganism is used as an ideal microbe to investigate the efficacy of different root canal irrigants and intra-canal drugs in endodontics. ${ }^{(10)}$ The selection of this microorganism in this study is also for the reasons mentioned above.

The success of root canal treatment depends on the ability of the irrigant to rinse and remove dentinal debris and to disinfect the root canal from microorganisms. ${ }^{(13)}$

Many irrigants are used in endodontic treatments, all of which have antimicrobial properties. (11) Desirable irrigant properties include the ability to dissolve pulp tissue, the ability to remove the smear layer, low toxicity, non-stimulation of adjacent tissues, and antimicrobial activity. ${ }^{(9)}$

Chemical debridement is needed, especially in teeth with complex internal anatomy where the root canal microorganisms cannot be completely cleansed by mechanical means alone. ${ }^{(14)}$

Sodium hypochlorite $(\mathrm{NaOCl})$ has been a common irrigant in endodontic treatments since it was first proposed in endodontics by Walker in $1936 .^{(14)}$

Different concentrations of $\mathrm{NaOCl}$ have been used in the last seven decades due to its good antimicrobial properties and its ability to dissolve pulp tissues. ${ }^{(15)}$

In a 2007 study, Giardino and colleagues investigated the antimicrobial effects of $\mathrm{NaOCl}$ and two other irrigants (MTAD and Tetraclean) on E. faecalis in the root canal system and found that 
only $5.25 \% \mathrm{NaOCl}$ can remove biofilm after five minutes. ${ }^{(15)}$ In 2009, Arias-Moliz and colleagues also found that $\mathrm{NaOCl}$ has a strong antimicrobial effect in the dental canal. ${ }^{(11)}$

Retamozo et al (2010) also investigated the antimicrobial effect of different concentrations of $\mathrm{NaOCl}$ and showed that $5.25 \% \mathrm{NaOCl}$ was more effective than $1.3 \%$ and $2.5 \%$ concentrations. $^{(9)}$

Therefore, in the present study, $5.25 \% \mathrm{NaOCl}$ was used, but since $\mathrm{NaOCl}$ has problems such as poor taste, lack of biocompatibility, high toxicity, corrosive properties, inability to remove the smear layer, and reduction of the elasticity coefficient and flexural strength of dentin, there are limitations to its frequent use in endodontic treatments. ${ }^{(16)}$ Given these limitations, attempts to find a substance with favorable $\mathrm{NaOCl}$ properties and fewer limitations are ongoing.

Another irrigant used in this study was $2 \%$ CHX. This irrigant contains 1,6-bis(4-Chlorophenyldiguanino)hexane digluconate and is used as a disinfectant in endodontics. ${ }^{(17)}$

$\mathrm{CHX}$ is an antimicrobial substance that affects a wide range of microbes, and its properties, such as water solubility, lower toxicity than $\mathrm{NaOCl}$, and its high stability, have led to the increasing use of this substance in endodontic therapies. ${ }^{(18,19)}$

In a 2004 study, Rosenthal and colleagues showed that the use of CHX as the final irrigant had a significant effect on reducing the number of microbes in the root canal system irrigated with $\mathrm{NaOCl}$ during treatment. ${ }^{(20)}$

Comparison of two dental canal irrigants, $\mathrm{NaOCl}$ and $\mathrm{CHX}$, yields different results:

In a 2008 study, Murray et al showed that $\mathrm{NaOCl}$ has higher antimicrobial power than $\mathrm{CHX} .{ }^{(21)}$

In a 2009 study, Williamson et al found $\mathrm{NaO}-$ $\mathrm{Cl}$ to be more effective than $\mathrm{CHX}$ in removing E. faecalis from the root canal system. ${ }^{(22)}$ These findings are in line with the results of the present study.

In a 2007 study by Oliveria et al, there was no significant difference between $\mathrm{CHX}$ and $\mathrm{NaOCl}$, ${ }^{(19)}$ which may be due to differences in bacterial assay methods.

However, the adverse effects of CHX, such as discoloration of teeth, loss of taste in some patients, mucosal burns, dry mouth, and discoloration of the tongue make us more likely to substitute other irrigants with more favorable properties. ${ }^{(21)}$ Herbal medications are one of the oldest results of human research. The use of herbal remedies due to their naturalness and fewer side effects has attracted the attention of many researchers. ${ }^{(6)}$

Today, researchers are seeking to replace chemicals with herbal and natural irrigants. ${ }^{(21,23)}$

In this study, we also investigated the antimicrobial effect of green tea extract as a dental canal irrigant. Green tea is the most common traditional drink in China and Japan, derived from young leaves of a plant called Camellia sinensis. EGCG is the most abundant polyphenol found in green tea, which in addition to cardiovascular reinforcement, its antioxidant and anticancer effects have also been proven in previous studies. Recently, the antibacterial effect of green tea and its polyphenolic elements on pathogenic and nonpathogenic microorganisms, such as Bacillus cereus, Acinetobacter baumannii, Mycobacterium, Helicobacter pylori, and gram-negative bacteria, has been demonstrated..$^{(6,24)}$

In a 2006 study, Hirasawa et al investigated the effect of green tea on the inhibition of acid production by dental plaque bacteria and demonstrated that the EGCG present in green tea is effective in reducing acid production in dental plaque by Streptococcus mutans. ${ }^{(25)}$

In a 2010 study, Prabhakar et al investigated the antimicrobial effect of green tea, MTAD, and $5 \% \mathrm{NaOCl}$, as root canal irrigants, on $\mathrm{E}$. faecalis. (7) They showed that $\mathrm{NaOCl}$ had the most prominent antimicrobial effect. In the cited study, there was a significant difference between green tea and $\mathrm{NaOCl}$, but Prabhakar and colleagues suggested that as the number of microorganisms naturally present in the root canal system is lower than the number they used in their study, this level of effectiveness of green tea in ex-vivo state is sufficient, and due to the side effects of $\mathrm{NaOCl}$ as well as easier access to natural products, low toxicity, reasonable price, and lack of persistent bacterial resistance to these substances, they suggested their use as an irrigant. They also recommended further research in this respect. ${ }^{(7)}$

In 2011, Pujar et al examined the antibacterial effect of green tea, Triphala, and $3 \% \mathrm{NaOCl}$ on E. faecalis in the root canal system. ${ }^{(16)}$ They also found a significant difference between green tea 
and Triphala with $3 \% \mathrm{NaOCl}$, but because of the acceptable antibacterial properties of green tea and Triphala and in the light of the adverse properties of $\mathrm{NaOCl}$, They recommended herbal alternatives to be used as irrigants. ${ }^{(16)}$

In this study, we also reached similar results. We investigated the antimicrobial effect of different concentrations of green tea extract with that of three other irrigants $(5.25 \% \mathrm{NaOCl}, \mathrm{CHX}$, and normal saline) on E. faecalis in root canal system. In this study, to mimic the clinical conditions, single-rooted and single canal human premolars without any internal or external resorption, calcification, caries and with closed root apices were used; these teeth have also been used in similar studies. ${ }^{(6,7,16,18)}$

The teeth were first cleaned of soft tissue debris using scaling curettes, and then, using a highspeed handpiece fitted with a tapered flat-end bur, an access cavity with direct access to the dental canal was prepared after caries removal. First, a \#10 K-file (Mani Inc., Tochigi, Japan,) was used to ensure apical patency, and the length was determined by the same file. The file was passed through the canal until it reached the apical foramen. Then, $1 \mathrm{~mm}$ was decreased from the length of the file to determine the working length; this method has also been used in other studies. ${ }^{(18,26)}$

Teeth with a working length of $16 \mathrm{~mm}$ were selected and then prepared using a rotary system with ProTaper files..$^{(7,18)}$

To equalize the conditions for all groups, the smear layer was removed by placing the teeth in $17 \%$ EDTA for one minute and then in 5.25\% $\mathrm{NaOCl}$ for four minutes. Finally, the teeth were rinsed with sterile distilled water for 10 minutes; this way, in addition to bacteria, the final irrigant also finds the chance to enter the dentinal tubules. ${ }^{(18,27)}$

In this study, the apical foramen of the teeth was sealed by light-cure glass-ionomer. To prevent external bacterial infiltration, the outer surface of the teeth was first coated with two layers of colorless nail polish and later with two coats of colored nail polish; the same method has been employed in similar studies. ${ }^{(8)}$

After sterilization by autoclaving and after bacterial inoculation, the samples were incubated at $37^{\circ} \mathrm{C}$ for two weeks. ${ }^{(7,9)}$

Next, to prepare different concentrations of green tea, the MIC of green tea extract was determined by serial dilution method and by determining the inhibition zone diameter against $\mathrm{E}$. faecalis (ATCC 29121), and finally, dilution of $31.25 \mathrm{mg} /$ $\mathrm{ml}$ of green tea extract was determined as the MIC. This method is a very accurate and valid method for MIC determination of essential oils and extracts, which has been used in other studies as well. ${ }^{(28)}$

According to the MIC determined for green tea extract, three concentrations of $3.125 \%, 12.5 \%$, and $25 \%$ were prepared.

In the next step, the canal was rinsed for 10 minutes by different irrigants. In the positive control group, the samples did not receive any treatment after the inoculation, and in the negative control group, the samples were sterilized only.

The laboratory model of this study has been used by other researchers. $(20,28)$ Of course, there are differences in some studies as well; for example, Prabhakar et al, in their 2010 study, immersed their specimens in irrigants, which is clinically impossible; therefore, the cited study cannot be considered reliable for clinical use. ${ }^{(7)}$

In a 2011 study by Pujar et al, after preparing the specimens and before irrigating them, the samples were cut vertically and then irrigated with the solutions, which is not clinically feasible, and the results cannot be generalized to clinical conditions. ${ }^{(16)}$

The procedure of sampling from the dental canals was postponed to the next day. Regarding the sampling method, we found some differences in different studies, for example, Ravanshad and colleagues sampled from inside the canal using \#45 paper points. ${ }^{(23)}$ Siqueira and colleagues did this in 1997 using paper points as well. (14) Prabhakar and colleagues sampled inside the canal using sterile paper points. ${ }^{(7)}$ Schäfer and Bössmann obtained dentin fragments using a Hedstrom file. ${ }^{(27)}$ In this study, for the sampling of the canals, we first removed the TSB fluid from the tooth canal using sterile paper points and added sterile physiological serum (equal to the volume of the canal) using a sterile 5-cc syringe with 18-gauge needles. After pouring the sterile serum, each canal was filed circumferentially with a Hedstrom file for 20 seconds. The file, along with sterile paper 
points used to collect serum in the canal (containing dentin fragments and possibly viable bacteria) was transferred into test tubes containing 10 cc of sterile normal saline, which were vortexed for 20 seconds to separate the surviving bacteria from the file and the paper points.

Next, for microbiological evaluation, the number of viable bacteria was calculated in CFU 48 hours later. This method has been used in other studies as well, and it has good accuracy and reliability due to the quantitative evaluation of bacterial reduction. ${ }^{(16)}$ However, in other studies, the criterion for microbiological evaluation has been the degree of turbidity, which is not an accurate criterion for evaluating the antimicrobial effect of a substance..$^{(9,14)}$

The results showed that there was a significant difference between $\mathrm{CFU}$ calculated for $\mathrm{NaOCl}$ and CHX compared to the CFU calculated for different green tea groups. The results showed the reduction in the number of microorganisms to be $100 \%$ with $\mathrm{NaOCl}, 98.9 \%$ with $\mathrm{CHX}, 58.35 \%$ with $3.125 \%$ green tea, $81 \%$ with $12.5 \%$ green tea, $94.8 \%$ with $25 \%$ green tea, and $57.5 \%$ with normal saline.

\section{Conclusion:}

Since the purpose of using irrigating solutions in root canal treatment is to disinfect the root canal rather than to sterilize it, the percentage of microorganism reduction observed for $12.5 \%$ and $25 \%$ green tea extract is acceptable. On the other hand, due to the naturalness of green tea extract, low toxicity, reasonable price, and lack of $\mathrm{NaOCl}$ and $\mathrm{CHX}$ side effects, green tea extract can be considered as a suitable alternative to conventional root canal irrigants. The results of this study showed that green tea extract at $12.5 \%$ and $25 \%$ concentrations reduced Enterococcus in the root canal.

\section{Acknowledgements:}

We would like to extend our gratitude and appreciation to all those who helped us with this project. No funding was received from any institution to conduct this research.

\section{References:}

1. Hahn CL, Liewehr FR. Relationships between caries bacteria, host responses, and clinical signs and symptoms of pulpitis. J Endod. 2007 Mar 1;33(3):213-9.

2. Sjögren U, Figdor D, Persson S, Sundqvist G. Influence of infection at the time of root filling on the outcome of endodontic treatment of teeth with apical periodontitis. Int Endod J. 1997 Sep 1;30(5):297-306

3. Gomes BP, Pinheiro ET, Jacinto RC, Zaia AA, Ferraz CC, Souza-Filho FJ. Microbial analysis of canals of root-filled teeth with periapical lesions using polymerase chain reaction. J Endod. 2008 May 1;34(5):537-40.

4. Torabinejad M, Khademi AA, Babagoli J, Cho Y, Johnson WB, Bozhilov K, et al. A new solution for the removal of the smear layer. J Endod. 2003 Mar 1;29(3):170-5

5. Wang CS, Arnold RR, Trope M, Teixeira FB. Clinical efficiency of $2 \%$ chlorhexidine gel in reducing intracanal bacteria. J Endod. 2007 Nov 1;33(11):1283-9.

6. Osterburg A, Gardner J, Hyon SH, Neely A, Babcock G. Highly antibiotic-resistant Acinetobacter baumannii clinical isolates are killed by the green tea polyphenol (-)-epigallocatechin3-gallate (EGCG). Clin Microbiol Infect. 2009 Apr 1;15(4):341-6.

7. Prabhakar J, Senthilkumar M, Priya MS, Mahalakshmi K, Sehgal PK, Sukumaran VG. Evaluation of antimicrobial efficacy of herbal alternatives (Triphala and green tea polyphenols), MTAD, and $5 \%$ sodium hypochlorite against Enterococcus faecalis biofilm formed on tooth substrate: an in vitro study. J Endod. 2010 Jan 1;36(1):83-6

8. Kakehashi S, Stanley HR, Fitzgerald RJ. The effects of surgical exposures of dental pulps in germ-free and conventional laboratory rats. Oral Surg Oral Med Oral Pathol. 1965 Sep 1;20(3):340-9.

9. Retamozo B, Shabahang S, Johnson N, Aprecio RM, Torabinejad M. Minimum contact time and concentration of sodium hypochlorite required to eliminate Enterococcus faecalis. J Endod. 2010 Mar 1;36(3):520-3.

10. de Paz LC. Redefining the persistent infection in root canals: possible role of biofilm communi- 
ties. J Endod. 2007 Jun 1;33(6):652-62.

11. Arias-Moliz MT, Ferrer-Luque CM, Espigares-García M, Baca P. Enterococcus faecalis biofilms eradication by root canal irrigants. J Endod. 2009 May 1;35(5):711-4.

12. Liu H, Wei X, Ling J, Wang W, Puang X. Biofilm formation capability of Enterococcus faecalis cells in starvation phase and its susceptibility to sodium hypochlorite. J Endod 2010;36:630-5. 13. Christensen CE, McNeal SF, Eleazer P. Effect of lowering the $\mathrm{pH}$ of sodium hypochlorite on dissolving tissue in vitro. J Endod. 2008 Apr $1 ; 34(4): 449-52$.

14. Siqueira JF, Machado AG, Silveira RM, Lopes HP, Uzeda MD. Evaluation of the effectiveness of sodium hypochlorite used with three irrigation methods in the elimination of Enterococcus faecalis from the root canal, in vitro. Int Endod J. 1997 Jul 1;30(4):279-82.

15. Giardino L, Ambu E, Savoldi E, Rimondini R, Cassanelli C, Debbia EA. Comparative evaluation of antimicrobial efficacy of sodium hypochlorite, MTAD, and Tetraclean against Enterococcus faecalis biofilm. J Endod. 2007 Jul $1 ; 33(7): 852-5$.

16. Pujar M, Patil C, Kadam A. Comparison of antimicrobial efficacy of Triphala, (GTP) Green tea polyphenols and 3\% of sodium hypochlorite on Enterococcus faecalis biofilms formed on tooth substrate: in vitro. J Int Oral Health. 2011;3(2):23-29.

17. Kishen A, Sum CP, Mathew S, Lim CT. Influence of irrigation regimens on the adherence of Enterococcus faecalis to root canal dentin. J Endod. 2008 Jul 1;34(7):850-4.

18. Bui TB, Baumgartner JC, Mitchell JC. Evaluation of the interaction between sodium hypochlorite and chlorhexidine gluconate and its effect on root dentin. J Endod. 2008 Feb 1;34(2):181-5.

19. Oliveira DP, Barbizam JV, Trope M, Teixeira FB. In vitro antibacterial efficacy of endodontic irrigants against Enterococcus faecalis. Oral Surg Oral Med Oral Pathol Oral Radiol Endod. 2007 May 1;103(5):702-6.

20. Rosenthal S, Spångberg L, Safavi K. Chlorhexidine substantivity in root canal dentin. Oral Surg Oral Med Oral Pathol Oral Radiol. 2004 Oct 1;98(4):488-92.

21. Murray PE, Farber RM, Namerow KN, Kuttler S, Garcia-Godoy F. Evaluation of Morinda citrifolia as an endodontic irrigant. J Endod. 2008 Jan 1;34(1):66-70.

22. Williamson AE, Cardon JW, Drake DR. Antimicrobial susceptibility of monoculture biofilms of clinical isolate of Enterococcus faecalis. J Endod. 2009;35:95-97.

23. Ravanshad S, Basiri E, Mohammadzadeh M. In vitro Evaluation of the Antimicrobial Effectiveness of Zataria multiflora as an Irrigant in Infected Root Canals with Enterococcus faecalis. J Dent (Shiraz). 2009;10(2):92-98.

24. Yang JC, Shun CT, Chien CT, Wang TH. Effective prevention and treatment of Helicobacter pylori infection using a combination of catechins and sialic acid in AGS cells and BALB/c mice. $\mathrm{J}$ Nutr. 2008;138(11):2084-90.

25. Hirasawa M, Takada K, Otake S. Inhibition of acid production in dental plaque bacteria by Green tea catechins. Caries Res. 2006;40(3);265270.

26. Nara A, Dhanu, Chandra P, Anandakrishna L, Dhananjaya. Comparative Evaluation of Antimicrobial Efficacy of MTAD, 3\% NaOCI and Propolis Against E Faecalis. Int J Clin Pediatr Dent. 2010 Jan-Apr;3(1):21-5.

27. Schäfer E, Bössmann K. Antimicrobial efficacy of chlorhexidine and two calcium hydroxide formulations against Enterococcus faecalis. J Endod. 2005 Jan;31(1):53-6.

28. Seghatoleslami S, Samadi N, Salehnia A, Azimi S. Antibacterial activity of endemic Satureja Khuzistanica Jamzad essential oil against oral pathogens. Iran Endod J. 2009 Winter;4(1):5-9.

Please cite this paper as:

Dadresanfar B, Vatanpour M, Farahmand M, Taheri S, Mahaseni Aghdam H. Ex Vivo Comparative Study of the Effect of Different Concentrations of Green Tea Extract and Two Common Irrigants on Root Canals Infected with Enterococcus faecalis. J Res Dentomaxillofac Sci. 2019; 4 (2) :32-40 